\title{
The Model of Social Innovation Project Evaluation
}

\author{
Tadeusz A. Grzeszczyk ${ }^{1,}$ a and Dariusz Klimek ${ }^{2, b}$ \\ ${ }^{1}$ Faculty of Management, Warsaw University of Technology, Poland \\ ${ }^{2}$ Faculty of Management and Production Engineering, Lodz University of Technology, Poland \\ atadeusz.grzeszczyk@pw.edu.pl, ${ }^{b}$ dariusz.klimek@p.lodz.pl
}

Keywords: Social innovation, Project management, Model of project evaluation, Developmental evaluation.

\begin{abstract}
The main aim of this paper is to discuss the model of evaluation of social innovation projects. At the beginning the theoretical background and foundations of social innovation are described. Then the essence of evaluation approaches that can be used in this field as well as multi-stage evaluation processes have been discussed. This information is the basis for presenting the model of evaluation of social innovation projects. The considerations are completed with an indication of possible directions for further research on new instruments useful in evaluation processes of such projects.
\end{abstract}

\section{Introduction}

Currently, intellectual discussions on the present and future of societies are underway, in which one of the main threads is to seek answers to research questions related to overcoming the most important challenges of our time. Therefore, it is necessary to improve the effectiveness of proposed and applied innovative thinking solutions and make it hard to implement a 'quantum leap' towards a truly sustainable future [1]. Such activities involve searching for answers to difficult questions and solving complex problems of evaluation of social innovation, which are most often implemented in the form of projects.

The implementation of any projects involves a significant risk of failure, which is particularly large in the case of innovative projects. This risk can be reduced thanks to appropriate evaluation models, methods and systems based on correctly selected smart indicators and criteria suitable for interdisciplinary projects.

Achievements to date in the discipline of management sciences, as well as in the field of project management largely focused on financial profits. It is advisable to develop research on social innovation projects, which are more difficult because they have usually vague, difficult to specify and multifaceted goals. It is worthwhile, therefore, to use the experience gained when running multifaceted projects and scientific achievements regarding the field of evaluation of different kinds of usually complex and many-sided evaluated projects: public, development and European [2].

The main aim of this paper is to discuss the model of evaluation of social innovation projects. The theoretical background and foundations of social innovation evaluation as well as multi-stage evaluation processes are presented in the beginning of the paper. Then the essence of evaluation approaches that can be implemented in this field has been discussed. Presented information is the basis for showing the model of evaluation of social innovation projects. The authors used the following research methods: literature analysis, non-participant observation and systems analysis.

\section{Social Innovation}

In the modern economy based on knowledge and innovations one can observe the dynamic development of new information and communication technologies as part of technological innovations regarding products and processes. Changes within society, incremental and radical innovations are rooted in new technologies development and their various applications, behaviour and beliefs of citizens as well as non-typical using of modern management approaches [3]. 
Positive developmental phenomena are accompanied by many social problems, such as professional and IT exclusion, elimination of certain jobs, poverty, homelessness, social inequalities, spotty quality of education, decreasing number of working age people (aging of societies), global climate change and others. Therefore, activities should be taken to ensure the regular development of technological innovations, as well as social innovations, in order to guarantee sustainable socio-economic development and constant improvement of people's quality of life at local, regional and global scales.

Social innovations are being undertaken to improve the socio-economic situation of countries, build social capital, obtain employment growth, activate individual people, local social groups and entire societies. This activation may concern, for example, teaching, training, shaping appropriate civic attitudes, implementation of educational processes in the spirit of respect for human rights and natural environment. Social reforms and innovations should be implemented both in the private and public sectors. Innovation is primarily about finding solutions that are more effective and efficient than those existing in a given area, and the effects are new values for individuals, groups and entire societies. The obtained results of social innovation projects, their impact on target groups should be evaluated using the basic evaluation criteria, first of all, efficiency and effectiveness.

Conducting research related to social innovation, it is worth combining them with the study on social capital to innovation in general and in other cases to social innovation research in particular. The relationships between social innovation and social capital can be showed at three following levels: the macro (some concept, in which social capital is as a driver of social innovation through lesson learned from community development projects and programs), the meso-organizational (the main role of social organizations, e.g. social enterprises) and the micro-individual (focusing on the social innovator role) [4].

Social capital is based on social cohesion, relations, networks, trust, cooperation, information-sharing and actions for the common good. In a macroeconomic perspective, social capital is a collective good which consist of trust-based networks that influence innovation activities and consequently economic growth rates of knowledge-based economies [5]. Various social innovation projects can positively or negatively affect the value of this capital which is related to enterprises with human, structural, fixed and market capitals (Figure 1).

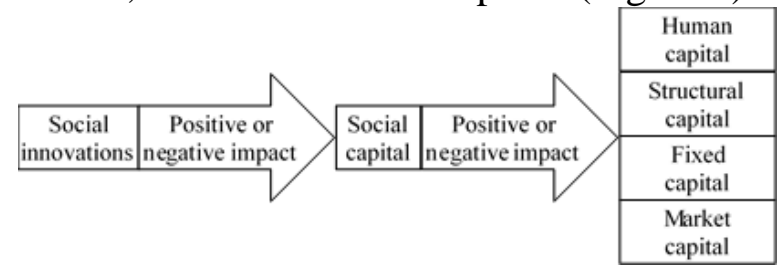

Fig.1. The potential impact of social innovations in enterprises (source: own study)

Complexity, multidimensionality and unstable conditions of many social problems require joint application in their analyzes of the systems approach, considerable creativity in the search for innovative solutions to solve these problems and modern approaches known from the dynamically developing field of evaluation. Implementation of social innovation projects evaluation is problematic, because project outcomes are relatively difficult to predict, especially in uncertainty conditions of frequent, dynamic, unpredictable and turbulent environment changes. The use of traditional evaluation methods that work well for other types of projects may not bring the expected results, producing different, unique, hard to interpret evaluation effects. Implementation of social innovation projects evaluation is a great challenge that can be met in the case of using the appropriate complex multi-aspect solutions.

In the case of evaluation of social innovation projects based on dynamic realities and complex environment, it is advisable to use models based on the systems thinking and developmental evaluation approach [6]. This approach can support innovation (in the form of products, projects, programs, organizational changes, complex system interventions etc.) and its evaluation through gathering and analyzing real time data to support ongoing decision making, serving design, development and implementation of processes [7]. Developmental evaluation is therefore 
fundamentally different from other types of evaluation approaches (e.g. formative, summative, etc.).

\section{Evaluation Approaches}

Evaluation can be presented as a systematic investigation concerning quality of projects, subprojects, programs, subprograms, and any of their elements or components which can be studied together or separately, for finding new knowledge, decision supporting, organizational development responding to the expectations of stakeholders, contributing to social and organizational value [8]. The theory and practice of evaluation are based on the achievements, approaches and methods known from economic sciences, management, social, political, public administration, legal and other. Interdisciplinary and systemic approaches should play an important role in this field.

Project evaluation should be presented as a multi-stage process in which there can, for example, occur the four following stages: structuring and designing some evaluation process, data gathering, collected data analysis, results formulation and suggesting recommendations. Various evaluation models, methods, techniques and tools can be allocated to individual stages of this process.

The basic models and methods belonging to the first stage, above all, are: selected representative from the family of logic models, Logical Framework Approach (LFA) based methods, Metaplan (shared understanding), classical SWOT analysis and others. At the data collection stage, one can, among other things, apply personal interview, survey, qualitative research, drop and collect survey, fieldwork observation, secondary data-based models and others. Collected data analysis applies, among others, various statistical analyzes, econometric models, comparative study between groups, shift analysis, mixed intelligent systems [9] and others. In particular, the use of solutions inspired mathematically and biologically can lead to significant advances in evaluation methods and models development [10]. The most popular models which support evaluation results formulation and suggest recommendations are usually based on multi-criteria expert estimations and statistical multi-criteria analysis. Other useful methods can include, above all, benchmarking, fragmentary cost-benefit analysis and cost-effectiveness analysis.

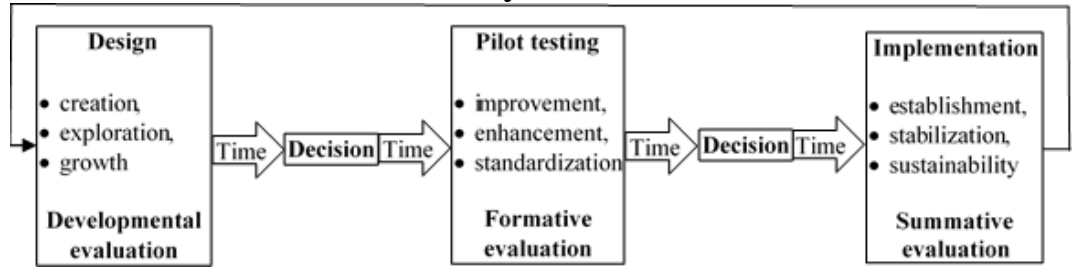

Fig. 2. Project life cycle and evaluation approaches (source: based on [11])

It is necessary to choose the right evaluation approaches for the relevant stages related to the project life cycle (Figure 2). At the stage of formulating concept and project design, developmental evaluation procedures are useful because they are particularly well-suited at the earliest stages of project life when social innovations are unclear and being formed. Pilot testing preceding the certain implementation is the stage of improvement, enhancement and standardization of project activities. The stage of implementation is related to the achievement of a certain maturity, the potential sustainability and predictability of project outcomes (short, medium and long-term) which can be studied using summative evaluation procedures.

Application of developmental evaluation supports and stimulates the creation of social innovation and raising public awareness during the implementation of evaluation processes in a flexible manner, adapted to the turbulent environment and dynamic changes of evaluated projects. In contrast to formative evaluation systems, developmental ones not only enable the formation and correction of evaluated projects, but also ensure self-correction.

\section{The Evaluation Model}

In the model, the three following evaluation approaches play a fundamental role: developmental, formative and summative (Figure 3). In accordance with the idea presented in the previous item of 
this paper, the first of these types is an important supplement to the second one and finds application in the initial phase of generating concepts of activities, defining the logical structure of proposed projects and their selection. Summative evaluation supports the formulation of conclusions concerning project outcomes obtained as a result of implemented processes. The combined occurrence and application of these three approaches in social innovation context is a fundamental requisite for productively conducting multifaceted research that regard social innovation evaluation [6].

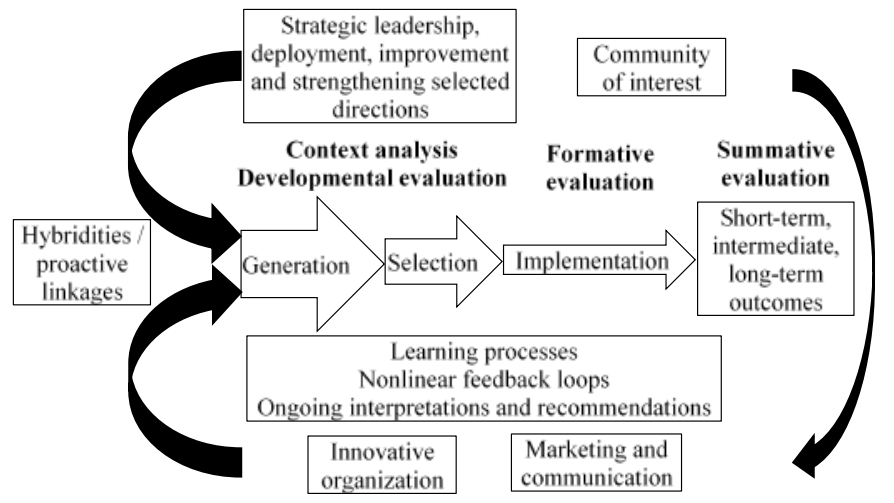

Fig. 3. Social innovation project evaluation (source: based on [12])

The model includes nonlinear feedback loops that are necessary because implemented social innovations can affect the functioning of whole organizations or their parts, not only once, but also gradually in the long term (in stages), modifying the previously achieved results of these innovations. In addition, the effects of social innovations in social, economic and environmental dimensions do not usually appear at the same time, and the results achieved in one dimension can shape effects in another one. It is also necessary to rely on continuous learning processes that can support the pursuit of an ongoing synthesis, formulation of interpretations and recommendations.

The presented model has a universal character and refers to various types of organizations. In the case of commercial organizations, it can be applied to large enterprises, which have sufficient financial resources to implement processes related to social innovation. Existing research results [13] prove that project management implemented in small and medium-sized enterprises is mainly focused on searching and acquiring financial resources and they lack the ability to carry out complex evaluation processes aimed at improving social innovation.

\section{Conclusion}

The model presented in this paper has some limitations and it is possible to specify the potential for its further development and enhancement. For example, the impact of social innovations on the value of social capital has not been clearly taken into consideration. It is important because this capital, which can be positively and negatively impacted by the effects of undertaken social innovations, has influence on other capitals of organizations, such as: human, structural, fixed and market. Evaluation of the impact of social innovations on social capital and, consequently, on other capitals, requires further research possibly leading to building another model, that would be an important complement to the one currently presented in this paper and would allow to build a more comprehensive and integrated approach to the evaluation of social innovation projects.

It is necessary to continue this type of research also regarding concretization of the suitable methods, systems and evaluation tools which can be used in accordance with this model. Social innovations are playing an increasingly important role in the development of modern societies and it is difficult to imagine effective solutions in this area without developing various types of evaluation instruments and models. In particular, crucial role in the progress of evaluation instruments should be played by concepts and theories existing beyond scientific disciplinary boundaries, applying systems and interdisciplinary ways of thinking as well as combined use of the three evaluation 
approaches: developmental, formative and summative.

Therefore, there is a need to develop comprehensive systems based both on the three evaluation approaches, as well as on the modification and combining of many methods from several specialized scientific disciplines that enable multi-aspect evaluation of social projects. This type of research methods should use latest achievements including, among other, social science, statistics, computing technologies, data science, machine learning, artificial intelligence and business decision theory, which can be used to develop new fragmentary and comprehensive methods.

\section{References}

[1] Thomas Osburg, Rene Schmidpeter (Eds.). Social Innovation: Solutions for a Sustainable Future [C]. Berlin Heidelberg: Springer-Verlag, 2013. 1-9.

[2] Tadeusz A. Grzeszczyk, Blazej Czajkowski. EU-funded Project Management in the Context of Suppliers and Contractors Selection [J]. Procedia Engineering, 2017. Vol. 182. 241-246.

[3] Marta Peris-Ortiz, Jaime Alonso Gomez, Patricia Marquez (Eds.). Strategies and Best Practices in Social Innovation: An Institutional Perspective [C]. Springer International Publishing AG, 2018. 1-10.

[4] Andre Habisch, Cristian R. Loza Adaui. A Social Capital Approach Towards Social Innovation [C]. Thomas Osburg, Rene Schmidpeter (Eds.) Social Innovation. CSR, Sustainability, Ethics \& Governance. Berlin, Heidelberg: Springer, 2013.

[5] Maria Thompson. Social capital, innovation and economic growth, Journal of Behavioral and Experimental Economics [J]. April 2018, Volume 73, 46-52.

[6] Peter Milley, Barbara Szijarto, Kate Svensson, J. Bradley Cousins. The evaluation of social innovation: A review and integration of the current empirical knowledge base [J]. Evaluation, 2018, Vol 24, Issue 2, 237 - 258.

[7] Michael Quinn Patton Developmental evaluation: Applying complexity concepts to enhance innovation and use [C]. New York, NY: The Guilford Press, 2011, 1.

[8] Donald B. Yarbrough, Lyn M. Shulha, Rodney K. Hopson, Flora A. Caruthers. The Program Evaluation Standards: A Guide for Evaluators and Evaluation Users [C]. Sage Publications, Thousand Oaks, CA 2011. XXV.

[9] Tadeusz A. Grzeszczyk. Mixed Intelligent Systems: Developing Models for Project Management and Evaluation [C]. Palgrave Macmillan/ Springer International Publishing AG, 2018.

[10]Tadeusz A. Grzeszczyk. Applications of Biologically Inspired Models in Project Evaluation [C]. Adam Jablonski (ed.), Business Models: Strategies, Impacts and Challenges, Nova Science Publishers, Inc, 2017. 241-248

[11]Hallie Preskill, Tanya Beer. Evaluating Social Innovation [C]. FSG and Center for Evaluation Innovation, 2012. 6.

[12] James Carr, Alfonso Molina-Fuenzalida, Eileen Wattam, Mark Backhaus. The REAL deal? The Application of a New Social Innovation Model [J]. 2011. 15, http://www.alfonsomolina.info/wp-content/uploads/2015/12/The-Application-of-a-New-SocialInnovation-Model.pdf (access: 06/2018).

[13]Remigiusz Kozlowski, Marek Matejun. Characteristic Features of Project Management in Small and Medium-Sized Enterprises [J]. E \& M Ekonomie a Management, 2016, vol. XIX, nr 1, 33-48. 